\title{
NEUTRON STUIDIES OF LIQUID AND SOLID HELIUM
}

\author{
PROGRESS REPORT
}

MAY 1,1986 - APRIL 30,1987

Henry E. Glyde

Department of Physics

University of Delaware

Newark, Delaware 19716

CONTENTS

I. Progress Report

II. References

III. Publications and presentations

Reprints of Pubilcations (Attached)

Prepared for

THE DEPARTMENT OF ENERGY

UNDER CONTRAC'T NO. DE-FG02-84ER45082

April, 1987

MASIER

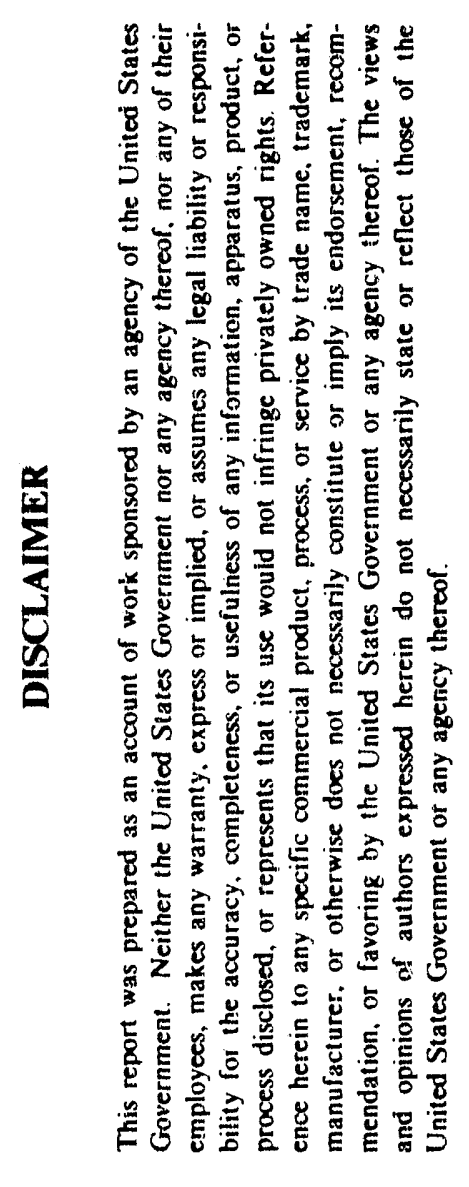




\section{SUMMIRY}

The progress made during $\$ / 1 / 86-4 / 31 / 87$ under contract No. DE-F902-34ER45082 is reported. The validity of the Impulse Approximation (IA) to the dynamic form factor. $S(Q, w)$, has been investigated using realistic models of solid helium. The calculations suggest that the IA can be used to obtain the momentum distribution, $n(p)$, within $1 \%$ at $Q \approx 30 \bar{A}^{-1}$, if $S(Q, w)$ is first symmetrized about the recoil frequency. wR. For solid helium under pressure (e.g. 5 kbar) a $Q 250 \dot{A}^{-1}$ is required. The $S(Q . \omega)$ in liquid $3 \mathrm{He}$ and $4 \mathrm{He}$ in the wave vector transfer range $3 \leq Q \leq 10 \mathrm{~A}^{-1}$ has been evaluated, beginning from the pair potential. The general shape and width of $S(Q, w)$ obtained agrees well with existing experiment. The width

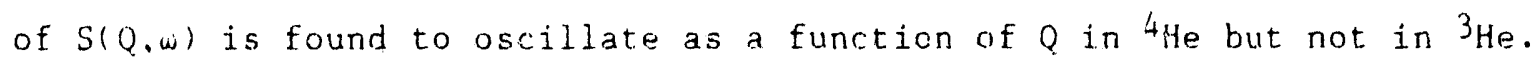
The dynamics of atoms adsorbed in solid lavers on surfaces has been studied using self-consistent methods. 


\section{PROGRESS REPORT}

Progress made during the period $5 / 1 / 86$ to $4 / 31 / 87$ is reported. Since a full proposal presenting the research proposed for $5 / 1 / 87-4 / 21 / 90$ was submitted in October 1980 , we present a progress report only here.

Progress has been made in thrpe broad areas: (1) Exploration of the validity of the Impulse Approximation. (2) Evaluation of kinetic energies in solid ${ }^{3}$ He and ${ }^{4}$ He, (3) Evaluation of the dynamic form factor, S(Q,w), in liquid ${ }^{3} \mathrm{He}$ and ${ }^{4} \mathrm{He}$ from first principals for $3 \leq Q \leq 15 \AA^{-1}$, and (4) evaluation of phonon energies and lifetimes in two-dimensional solids. This is presented in sections $1-4$ below.

\section{IMPULEE APPROXIMATION}

In the impulse approximation (IA), $S(Q, \omega)$ is approximated by

$$
S_{I A}(Q, w)=\int d \vec{p} n(\vec{p}) \delta\left(w-w_{R}-\frac{\vec{Q} \cdot \vec{p}}{M}\right)
$$

where $u_{R}=h Q^{2} / 2 M$ is the recoil frequency and $n(\vec{p})$ is the equilibrium fraction of atoms having momentum in the range $\vec{p}$ to $\vec{p}+d \vec{p}$. When the IA holds (with reasonable accuracy) the $n(\vec{p})$ can be extracted from an observed $S(Q, w)$. in the context of helium the IA was first mentioned by Miller et all and articulated by Hohenberg and Platzman. 2 The aim was to 
obtain the condensate fraction $n_{0}$ in liquid ${ }^{4}$ He from $S(Q, w)$. It has been used primarily for this purpose $e^{3-9}$ plus determinations of $n(p)$ in liquid ${ }^{4}$ He and 3 He by Martel et al 9 and Mook ${ }^{10}$, respectively. The term impulse approximation appears to have been coined by chewll in the context of proton scattering from deuterons, although it has been used in Compton scattering 12.13 from electrons since the late 1920 's. With the advent of pulsed neutron sources, the IA will become increasingly employed in helium.

The validity of the IA has enjoyed a lively debate.8,14-23 It will approximate $S(Q, \omega)$ at high $Q$ when the impulse, $h Q$, transferred from the neutron to the scattered atom is much greater than the impulses transferred to the scattered atom from its neighbors. For atoms interacting via a smooth protential the IA is exact $7,14,23$ for $Q \rightarrow \infty$. However, for atoms interacting via potentials having a perfectly hard core le.g. a fluid of hard spheres) the $I A$ is never reached $15-17$ even at $Q \rightarrow \infty$. In hard sphere collisions, the impulses transferred between atoms, Fr, where $F \sim \delta V / \delta x$, can be infinite no matter how short a time scale $\tau$ we consider. Helium atoms interact via a smooth potential which is steeply repulsive (but not infinitely steep). Thus we expect the IA to hold at $Q \rightarrow \infty$. The questions are; how high a $Q$ is required for the IA to hold with sufficient accuracy in helium to be useful? What are the most accurate methods for determining $n(p) ?$

In this context we undertook calculations of $S_{i}(Q, w)$ (the incoherent dynamic form factor) for a realistic morel of solid helium.20,21,24 The aim was to compare $S_{i}$ and $S_{1 A}$ for the same model. We assumed that $Q$ is large enough that the interatomic interference terms in $S(Q, w)$ may be 
neglected and $S(Q, \omega)$ reduces to $S_{i}(Q, \omega)\left(Q \geq 10 A^{-1}\right.$ in helium). The difference

$$
\delta S=S_{i}(Q, w)-S_{I A}(Q, i)
$$

provides a direct measure of the departure of $S$ from $S_{I A}$ due to "final state interactions".

Solid helium was considered sinc. microscopic models of $S(Q),(w)$, beginning with the pair interatomic potential, can be made. The microsopic model was a self-consistent phonon theory coupled with a T-matrix treatment of the steeply repulsive core. From the phonon energies and lifetimes an anharmonic density of states, $g(i)$, was computed. This $g(w)$ sets the energy scale of the solid. It also has an anharmonic high frequency tail due to the core of the interatomic potential.

The $S_{i}(Q, t)$, where the 1-2 phonon interference terms vanish, is well approximated at $\mathrm{T}=0 \mathrm{~K}$ by

$$
S_{i}(Q, \omega)=\frac{1}{2 \pi} \int d t e^{i \omega t} e^{-2 W} e^{i j} R \int_{0}^{\infty} d w^{\prime} g\left(w^{\prime}\right) \frac{1}{w^{\prime}} e^{-i w^{\prime} t}
$$

The IA is fundamentally a high energy transfer, short time approximation. It may be obtained from (2) by approximating $e^{-i \omega t}=1-i \omega t-\$ \omega^{2} t^{2}$ (short time approximation) giving,

$$
S_{I A}(Q, w)=\left(2 \pi \sigma^{2}\right)^{-\frac{2}{2}} e^{-\frac{1}{2}(\omega-\omega} R^{2} / \sigma^{2}
$$




$$
-6-
$$

where $\sigma^{2}=\omega_{R}\left\langle\omega^{1}\right\rangle=\omega_{R}(4 / 3 \hbar\rangle\langle K E\rangle$. Here $\left\langle\omega^{n}\right\rangle$ is the $n^{\text {th }}$ moment of $g(\omega)$ and $\langle K E\rangle$ is the atomic kinetic energy (strictly the zero point energy here). The IA in this case is a Gaussian because we have assumed statistically independent phonons.
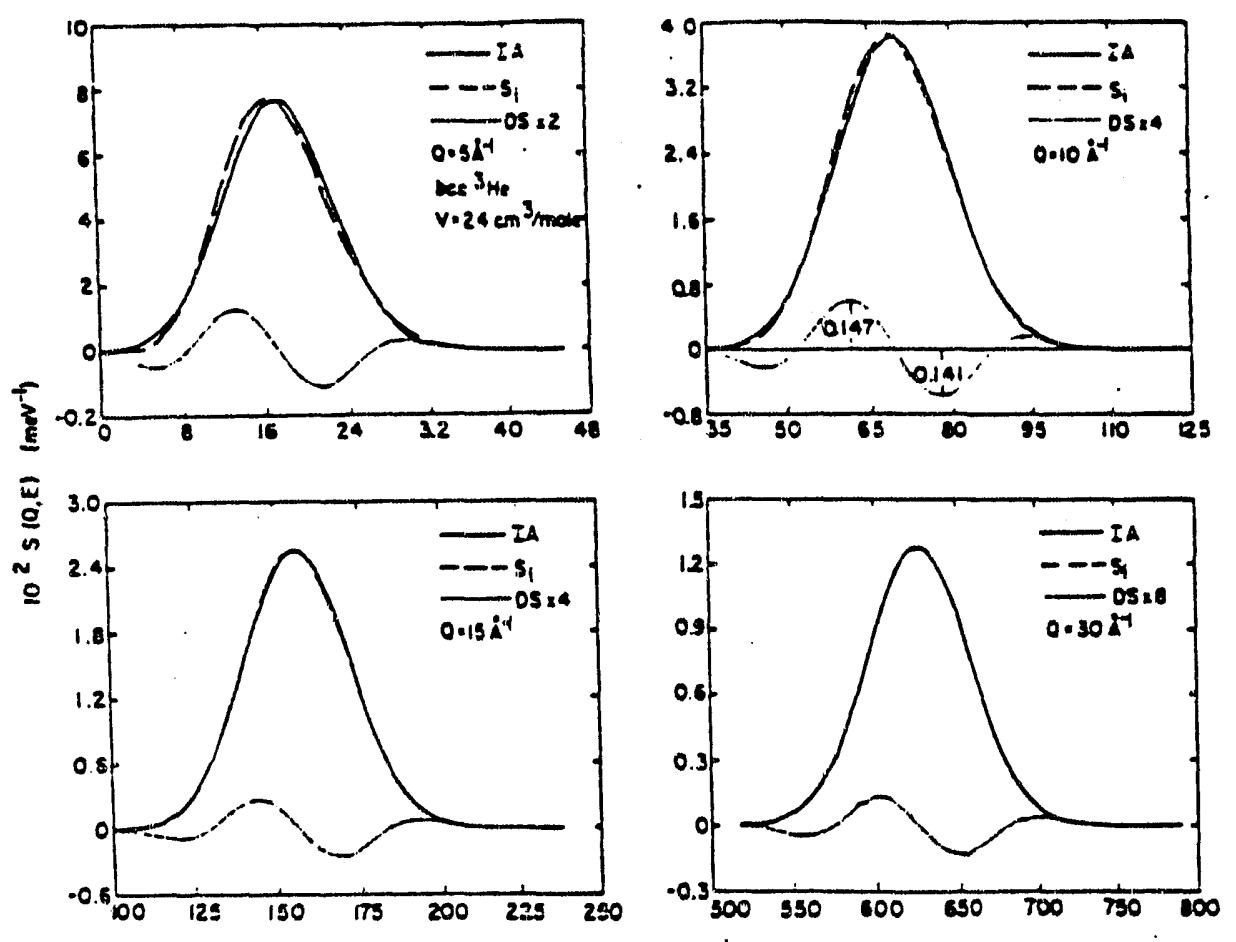

Fig. 1

Fig. 1: Incoherent, $S_{i}(Q, \omega) ; I A, S_{I A}(Q, \omega) ; D S=S_{i}-S_{I A}$. (From Glyde, Ref. 20).

In Fig. 1 we show $S_{i}, S_{I A}$ and $\delta S=D S$ for bcc ${ }^{3}$ He. The $\delta S=0$ at $\omega=$ $\omega_{R}$ and is nearly anti-symmetric in $\left(\omega-\omega_{R}\right)$. The leading term in $\delta S$ (obtained by keeping the $t^{3}$ term in the expansion of $e^{-i \omega t}$ ) is 23 


$$
\begin{aligned}
\delta S_{1}(Q, \omega) & =-\frac{\left\langle\omega^{2}\right\rangle}{\left\langle\omega^{2}\right\rangle} \frac{(\omega-\omega)^{2}}{2}\left[1-\frac{\left(\omega-\omega_{R}\right)^{2}}{3 \sigma^{2}}\right] S_{I A}(Q, \omega) \\
& =-\frac{\left\langle(\omega)^{2}\right\rangle}{\left\langle{ }^{1}\right\rangle} \frac{v}{Q}\left[1-\frac{E_{v}}{\langle K E\rangle}\right] S_{I A}(y)
\end{aligned}
$$

where $y=(M / Q)(\omega-\omega R)$ is the West 25 or $y$-scaling variable and $E_{y}=h^{2} y^{2} / 2 M$. The $\delta S_{1}$ is clearly anti-symmetric about "N suggesting that $\delta S$ is well approximated by $\delta S_{1}$. Clearly if the higher moments $\langle\omega n\rangle$ are large, then high $Q$ will be required to reach the IA.

Also by comparing $\delta S$ and $S_{I A}$ at $Q=15$ and $30 \dot{A}^{-1}$ in Fig. 1 we see that $\delta S$ scales as $1 / Q$ relative to $S_{I A}$. as suggested by (4). This shows that $\delta S$ is dominated by the $\delta S_{1}$ at $? \geqslant 30 \AA$. approaches $S_{I A}$ as $Q$ increases for a realistic model of solid helium.

To determine how accurately $S_{i}$ approaches $S_{I A}$, we 21.24 have compared the momentum distribution, $n(\vec{p})$, obtained from $S_{j}(Q, w)$ with the true $n(\vec{p})$. From $(1)$,

$$
n(\vec{p})=\left.\mp \frac{1}{2 \pi p}\left(\frac{Q}{M}\right)^{2} \frac{\partial S_{I A}(Q, \omega)}{\partial \omega}\right|_{\omega=\omega_{R}} \pm \frac{p Q}{M} .
$$

When $S_{i}(Q, w)$ is substituted into (5), an approximate or different function $n_{u}(\vec{p})$ is obtained. As $s_{i} \rightarrow S_{I A}, n_{u} \rightarrow n$. We found 21 that although $s_{i}$ looks like SIA on a graph, say at $Q=30 \bar{A}^{-1}$, the $n_{U}$ and $n$ differ by $\sim 10 \%$ at $Q=30 \dot{A}^{-1}$. The discrepancy between $n_{u}$ and $n$ can be reduced by a factor of 10 using the symmetrization procedure discussed by Sears. $7,9,14$ This 
procedure eliminates the antisymmetric term $\delta S_{1}$ in (4) from $S_{i}$. In (5), if. we use the upper (lower) sign, we evaluate the derivative on the right (left) of $w_{R}$. If we average the two $n_{u}(\vec{p})$ evaluated with upper and lower signs, we obtain a symmetrized $n_{s}(\vec{p})$. This symmetrized $n_{s}(\vec{p})$ is a much improved approximation to $n(\vec{p})$. Indeed in $(5)$ when $S_{i}=S_{I A}$ and $S_{i}$ is not symmetric about $\omega_{R}$, we do not know wich sign to use in (5). We therefore conclude that $S_{i} \rightarrow S_{I A}$ at high $Q$ in solid helium. Using a symmetrizing procedure the $n_{s}(p)$ obtained from $S_{i}$ approaches the true $n(p)$ within $1 \%$ in solid ${ }^{4} \mathrm{He}$ at svp for $Q \sim 30 \mathrm{~A}^{-1}$. The $S_{i}(Q, w)$ in bec ${ }^{3} \mathrm{He}$ also provide functions that can be compared directly with experiment to test the self-consistent phonon theory and different models of short range correlations.

\section{Kinetic Energies in Quantum Solids}

The kinetic energy of atoms in solid helium is suprisingly large. This is due directly to the highly anharmonic nature of quantum solids. To show this and to compare with experiment, we 26 have calculated $\langle k E\rangle$ in bcc ${ }^{4} \mathrm{He}$ and ${ }^{3} \mathrm{He}$.

Firstly, if we assume srilid helium is a moderately anharmonic solid, we may estimate the $\langle\mathrm{KE}\rangle$ using a Debye model. The Debye temperature, ${ }_{0} \mathrm{D}$, could be adjusted to fit the observed disperison curves or the observed Debye-waller factor. In the Debye model, $\langle K E\rangle=(9 / 8) \theta_{0}$. This yields a 〈KE〉 which is approximately $50 \%$ of the observed value. Similarly, we might 
assume a Gaussian vibrational distribution for the atoms around their lattice points. The width of this distribution can be determined to fit the observed Debye-Waller factor. Again with this model a 〈KE the observed value is obtained. The Debye $\langle K E\rangle$ is compared with the observed values and values calculated using Monte Carlo (MC) methods in Fig. 2. The large $\langle K E\rangle$ of solid helium is a clear signature of the highly anharnonic nature of solid helium.

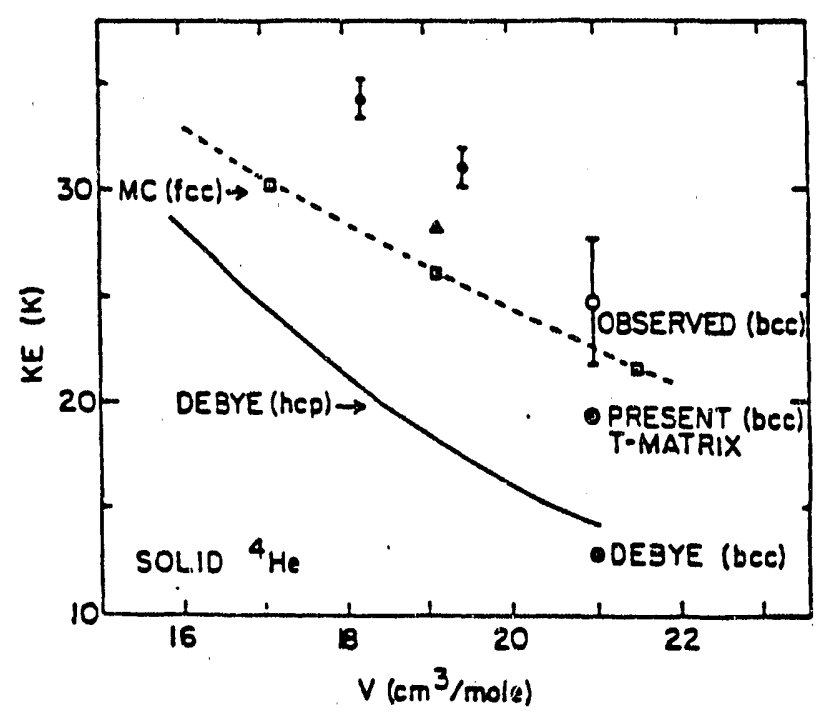

Fig. 2

Fig. 2: 〈KE〉 in solid ${ }^{4} \mathrm{He}: \zeta$ and $\zeta$, observed; $D$ and $\Delta$, Monte Carlo (MC). (From Moleko and Glyde, Ref. 26).

The $\langle K E\rangle$ may be evaluated using the full, anharmonic one-phonon response function $A(q \lambda, \omega)$. This function has talls reaching up to high $w$ well above the mean one-phonon frequency $\omega_{q} \lambda$, especially for longitudinal phonons for $q$ near the Brillouin zone edge. These high energy (ho) tails arise from the short lifetime of the phonons. The tails are due ultimately 
to the steeply repulsive (anharmonic) potential. The $\langle\mathrm{KE}\rangle$ in terms of the $A(q \lambda, w)$ is

$$
\langle k E\rangle=\frac{\hbar}{4 N} \sum_{q \lambda} \frac{1}{\omega_{q} \lambda} \int d \omega \omega^{2} A(q \lambda, \omega) .
$$

The presence of the (1) 2 in (7) means that the tails of $A(q \lambda, v)$ contribute significantly to $\langle\mathrm{KE}\rangle$. On the other hand the mean square vibrational ampliltude $\left\langle u^{2}\right\rangle$ appearing in the Debve-waller factor is

$$
\left\langle u^{2}\right\rangle=\frac{\hbar}{2 N M} \sum_{q \lambda} \int d w A(q \lambda, w)
$$

so that the tails of $A(q \lambda, w)$ do not contribute greatly to $2 W=\left\langle[Q \cdot u]^{2}\right\rangle$. Using a microscopic model (T-matrix and self-consistent phonons) we have calculated the $\langle K E\rangle$ in bcc ${ }^{4} \mathrm{He}$ and obtained a value which lies somewhat below but in reasonable agreement with experiment (see Fig. 2). In this way we may calculate phonon energies, $\left\langle u^{2}\right\rangle$ and $\langle K E\rangle$ in reasonable agreement with experiment. This is pnssible only if $A(q \lambda,(w)$ has high energy tails. As has been noted previously, 16,24-30 this demonstrates the existance cf high frequency tails in $S(Q, W)$.

We have also evaluated the $\langle K E\rangle$ in bcc ${ }^{3} \mathrm{He}$ at several volumes. As expected, the anharmonic 〈KE〉 is much larger than predicted by a Debyemodel. The volume dependence of the anharmonic $\langle K E\rangle$ is also smaller than predicted by an approximately harmonic or Debye model. These 〈KE〉 values have been communicated to Professor R. O. Sirnmons who is planning a 
measurement at Argonne National Laboratory. These measurements in bcc ${ }^{3} \mathrm{He}$ can distinguish between different models of the dynamics of solid He.

\section{Excitations in Liquid 3 He at High Q}

We have undertaken a general study of excitations and $S(Q, \omega)$ in liquid $3_{\text {He }}$ at high momentum; $4 \leq Q \lesssim 15 \AA^{-1}$. The aim is to evaluate $S(Q, w)$ for comparison with the observed values of Mook 10 and of Sokol et al ${ }^{31}$ and to compare wilh experiments in progress at Argonne National Laboratory.

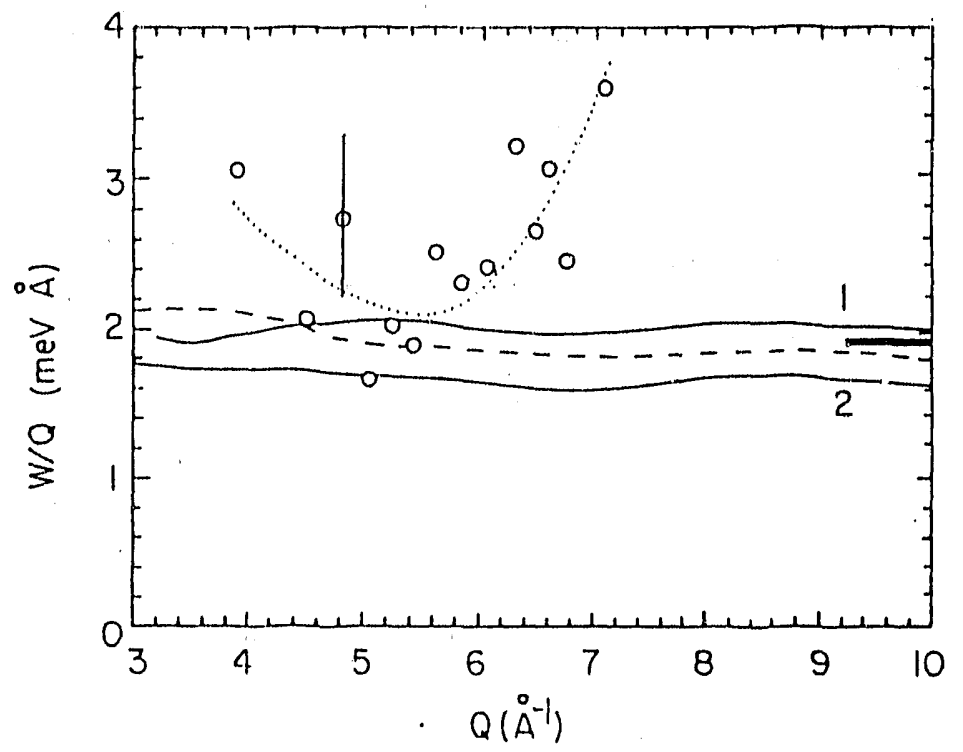

Fig. 3

Fig. 3: Widths of $S(Q, w)$ in liquid ${ }^{3} \mathrm{He}(0)$ Mook's data and gujde to eye $(\ldots \ldots),($ ) present calculations using models 1 and 2 , ( $\longrightarrow$ Sokol et al.'s observed $W(Q) / Q$ for $12 \leq Q \leq 15 \AA^{-1}$.

There are two issues of special interest. The first is the Full width at Half Maximum, $W(Q)$, of $S(Q, \omega)$ as a function of $Q$. Mook 10 has measured 
$W(Q)$ in the range $3 \leq Q \leq 7 \AA^{-1}$. He finds an average value of $W(Q) / Q=$ $\therefore .18$ meV $\AA$ and that $W(Q)$ has a minimum at $Q \approx 5.5 \AA$ (see Fig. 3). Sokol et al also find $W / Q \approx 1.9 \pm 0.30 \mathrm{meV} \AA$ at $12 \leq Q \leq 15 \AA^{-1}$. In liquid $4 \mathrm{He}$, Martel et al ${ }^{9}$ and Cowley and Woods ${ }^{32}$ observe that the FWHM, W(Q), in ${ }^{4} \mathrm{He}$ "oscillates" with Q (see Fig. 4). In the IA, $W(Q) / Q$ is a constant.

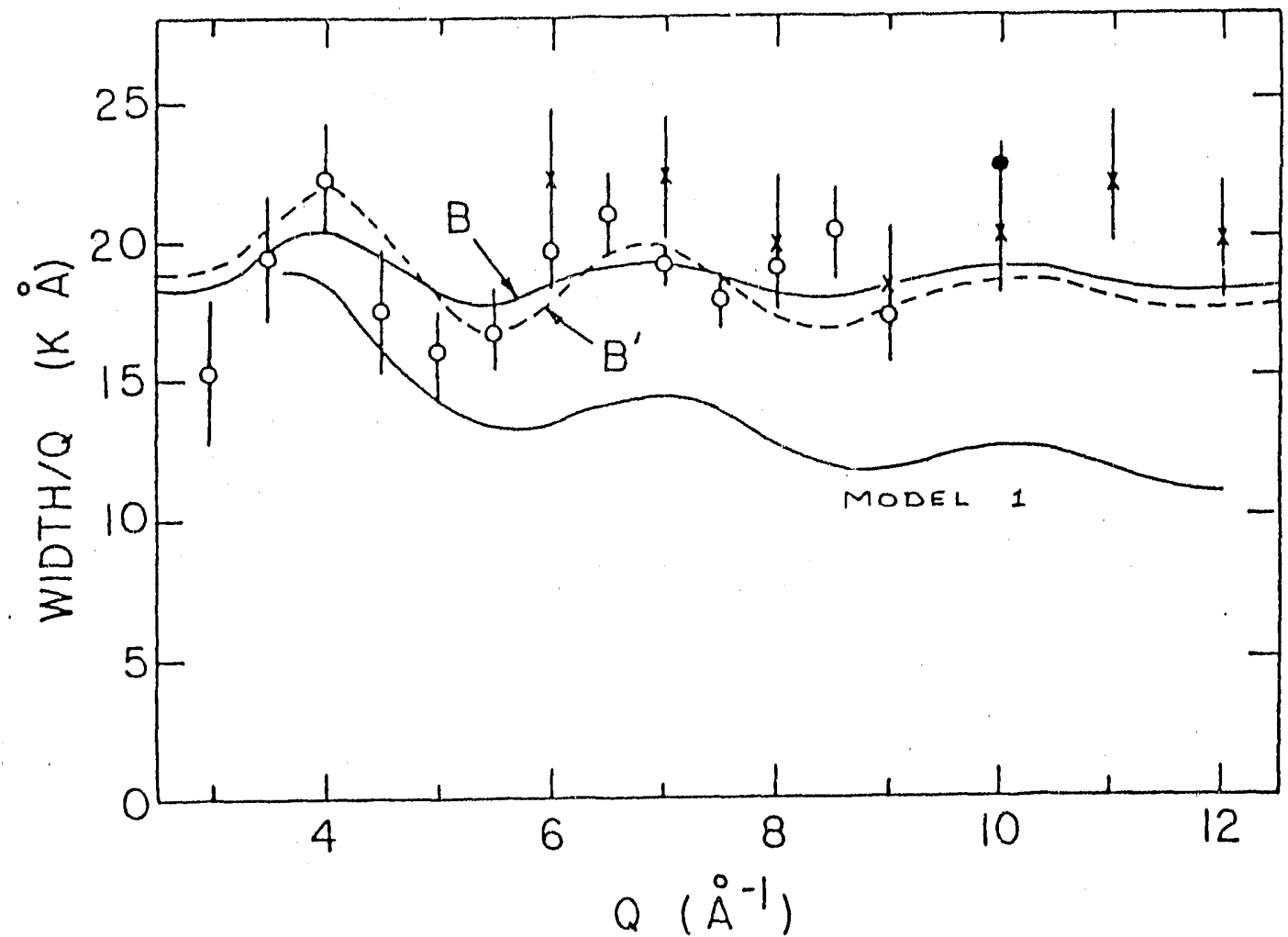

Fig. 4

Fig. 4: Widths of $S(Q, \omega)$ in liquid ${ }^{4} \mathrm{He}:(0)$ and $(x)$ data of Martel et al. (Ref. 9); B and B' model calculations from Ref. 9; present width using model 1 . 
Martel et al have suggested that the oscillations in w(Q) are related to oscillations in the HewHe atom scatrering cross-section $\sigma(Q)$. For exampie. 25 the $\sigma(Q)$ for two free He atoms scattering in free space is shown in Fig. 5. The $\sigma(Q)$ indeed ostillates with $Q$. The oscillations in the ${ }^{3}$ He- ${ }^{3}$ He $\sigma(0)$ are nearly $180^{\circ}$ "out of phase" with those in the 4He-4He $\sigma(0)$. Martel et al drew the connection between $S(Q . w)$ and $\sigma(Q)$ using a simple model.

$$
\operatorname{sic}, w)=\frac{1}{R} \sum_{p} n(p) \frac{1}{\pi}\left[\frac{\gamma(p+Q)}{\left(w-w_{k}-\frac{\vec{p} \cdot \vec{Q}}{M}\right)^{2}+r(p+Q)}\right]
$$

in which

$$
\gamma(Q)=n\left(\frac{h Q}{n^{2}}\right)(Q)
$$

The relation (3) was proposed by Hohenberg and Platzman.2 It may be derived using kinetic theory arguments. These arguments give the lifetime $\Gamma=T^{-1}=n v \sigma$ for a particle of velocity $v=n Q / M$ in a dilute gas of density $n$ due to inter-particle collisirs. Using (B) with a Gaussian $n(p)$ Having ( $p^{2}$; adjusted to fit experiment Marte) al obtained the line shown as $B$ in Fig. 4 which reproduced their observed $W(Q)$ quite well. A goal here is to evaluate $\mathrm{W}(\mathrm{Q})$ in liquid ${ }^{3} \mathrm{He}$ from first principles to see whether the observed $w(Q)$ can be obtained and to investigate the origins of the variation of $h(Q)$ with Q. Particularly, if (9) is correct, W(Q) should also oscillate with? in 3he. 


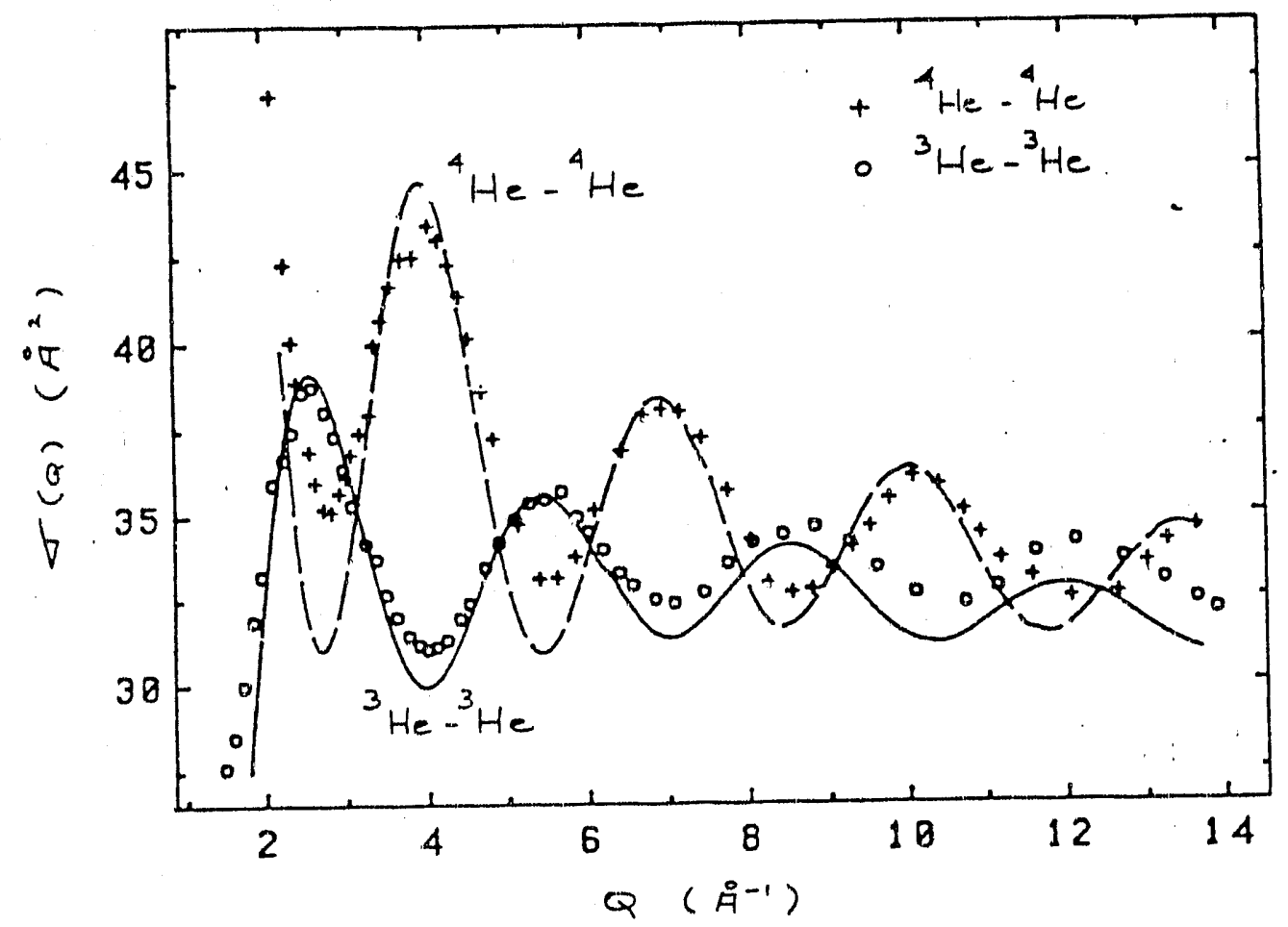

Fig. 5

Fig. 5: He-He atom cross-section: $+\left({ }^{4} \mathrm{He}\right)$, o( $\left.{ }^{3} \mathrm{He}\right)$ observed, Feltgen et al., J. Chem. Phys. 76, 2360 (1982); present calculations.

Secondly, the atomic kinetic energy is related to the second moment of the incoherent $S_{j}(Q, w)$ by

$$
\sigma^{2}=\int d_{\omega}\left(\omega-\omega_{R}\right)^{2} S_{j}(Q, \omega)=\frac{4}{3 \hbar} \omega_{R}\langle K E\rangle \text {. }
$$

If $S_{i}(Q, \omega)$ is assumed to have a Gaussian shape, then the $\langle K E\rangle$ is related to $w(Q)$ by 


$$
\langle K E\rangle=\frac{3}{32 \ln 2}\left(\frac{\hbar^{2}}{2 M}\right)^{-1}\left(\frac{W}{Q}\right)^{2} .
$$

This relation was used by Mook ${ }^{10}$ and Sokol et a $1^{31}$ to obtain the $\langle k E\rangle$ from their observed values of $W$. A second immediate goal is to investigate the shape of S(Q,w), to evaluate $\sigma^{2}$ directly and to test (11).

Our calculation of $S(Q, w)$ is a first principles calculation beginning from the pair He-He potential. We use the form proposed oy Aziz et al. 32 The properties of liquid ${ }^{3}$ He are then evaluated using the GaiitskiiFeynman-Hartree-Fock approximation. 46 This method describes the "dressed" pair interaction (particularly the repulsive core) in the liquici accurately. This interaction is the Galitski-Fevnman (GF) T-matrix interaction. It ignores the interation between a pair of atoms via the collective excitations. Since collective excitations disappear at $Q \geq$ $2 \hat{A}^{-1}$, the T-matrix should describe the full pair interaction well at high Q. Using the T-matrix, the energies and lifetimes of single particles $E(p)$ having momentum $p$ are evaluated within the Hartree-Fock (HF) approximation. This GFHF theory forms the basis of our calculation of $S(Q, w)$. $S(Q, w)$ is related to the dynamic susceptibility $Y(Q, w)$ ry

$$
S(Q, w)=-\frac{1}{n \pi} \operatorname{Im} \chi(Q, w) .
$$

In general, $(Q, w)$ is related to the interparticle interaction $\Gamma$ in the fluid by a complicated integral equation. At high $\left.Q(Q)>P_{F}\right)$, where $P_{F} \sim$ $0.8 \dot{A}^{-1}$ is the Fermi momentum) we assume that $\Gamma$ depends chiefly on $Q$ (and $w$ ) 
and much less on the momentum $p$ of the particles involved in the interaction. In this case the equation for $x$ reduces to the RPA result,

$$
X(Q, w)=\frac{X_{0}(Q, \omega)}{1-\Gamma(Q, w) Y_{0}(Q, w)}
$$

where

$$
Y_{0}(Q, w)=\frac{1}{\Omega} \sum_{p} \frac{n(p)-n(p+Q)}{w-\varepsilon(p+Q)+\varepsilon(p)+i n} .
$$

Here $\Gamma(Q, w)$ is taken as the T-matrix interaction, $E(p)$ is the single particle energy, $\mathrm{n}(\mathrm{p})$ is the momentum distribution and $x_{0}$ is the Lindhard function.

We have considered two models:

Model 1

To test the relation between $W(Q)$ and $\sigma(Q)$ we consider a very simple model consisting of two approximations applied to (13) and (14). Firstly, the $\Gamma(Q, w)$ is assumed to be the t-matrix or scattering amplitude of two atoms in free space, which we denote as $\Gamma(Q)$. From this $\Gamma^{O}(Q)$ we may calculate o(Q). Our calculated o(Q) is shown in Fig. 5 and clearly agrees with the observed values. The difference between o(Q) for ${ }^{3} \mathrm{He}-{ }^{3} \mathrm{He}$ and ${ }^{4} \mathrm{He}-{ }^{4} \mathrm{He}$ is only in the angular momentum (L) components which must be selected to satisfy statistics. At $Q \sim 10 \AA^{-1}$, we found 15 L components of $\Gamma^{\circ}(0)$ were needed to calculate $\Gamma^{\circ}$ accurately. Secondly, free particle energies were used in (14). Thus, in summary, we use (13) and (14) with 
$\Gamma(Q, w) \rightarrow \Gamma O(Q)$

(Model 1)

$$
\varepsilon(p) \rightarrow \varepsilon^{o}(p)=p^{2} / 2 M
$$

The FWHM, $W(Q)$, of S(Q,w) in Model 1 is shown in Fig. 3 as line 1 . The value of $W(Q)$ is in good agreement with observed values. However, $W(Q) / Q$ is essentially independent of $Q$. Thus although $\Gamma O(Q)$ (and $\sigma(Q)$ ) oscillates with $Q$ the $W(Q)$ does not oscillate with $Q$. Thus oscillations in $W(Q)$ and o(0) are not necessarily simply related - as suggested by (9).

\section{Model :}

Here we use the GFHF theory outlined above to calculate S(Q, (i) . The $\Gamma(Q$, (i) $)$ is the GF T-matrix for the scattering of two ${ }^{3}$ He atoms in liquid ${ }^{3}$ He. It includes the effects of the fermi surface and the renormalized (and complex) single particle energies $\varepsilon(p)=\varepsilon O(p)+\varepsilon\left(p, \varepsilon_{p}\right)$. In $x_{0}$ we also use the GFHF $E(p)$. Particularly important is the imaginary part of $\sum$. Thus

$$
\begin{aligned}
& \Gamma(Q, w)=\Gamma(Q, w) \\
& \varepsilon(p)=\frac{\Gamma^{2}}{2 M}+\Sigma\left(p, \varepsilon_{p}\right)
\end{aligned}
$$

In Fig. 3 we show W(Q) calculated using Model 2 as line 2 . The predicted $w(0) / Q$ again has only very small amplitude oscillations with $A$, too small to be objerved. Thus, models 1 and 2 do not predict observable oscillations in $W(0) / 0$ in ${ }^{3} \mathrm{He}$. 
We have also used model 1 to evaluate $W(Q) / Q$ in liquid ${ }^{4}$ He. The result is shown as the solid line marked MODEL 1 in Fig. 4. There we see that model 1 predicts oscillations $W(Q) / Q$ of magnitude, period and phase which agree well with the observed oscillations. These oscillations come directly from oscillations in the T-matrix scattering amplitude in the RPA, Eq. (13). The magnitude of $W(Q) / Q$ lies somewhat below the observed value, and decreases with $Q$, because we have used a free particle momentum distribution in model 1 .

Thus our model predicts oscillations in $W(Q) / Q$ in ${ }^{4}$ He but very small or no oscillations in 3 He. We believe this arises from a combination of two physical effects. Firstly, the magnitude of $W(Q)$ is larger in ${ }^{3}$ He than in ${ }^{4} \mathrm{He} ;\left(W(Q) / Q \approx 22-24 \mathrm{~K}\right.$ in ${ }^{3} \mathrm{He}$ versus $W(Q) / Q \approx 18-22$ in ${ }^{4} \mathrm{He}$. Thus the "Doppler" width due to kinetic effects is larger in 3 He than in 4 He, due to the larger zero point energy of 3 He. This larger Doppler width tends to mask or dominate variations in the width due to interactions or final state effects. Secondly, the oscillations in the T-matrix scattering amplitude is smaller for ${ }^{3}$ He than for ${ }^{4}$ He. This is reflected, for example, in smaller amplitude of the oscillations of the ${ }^{3} \mathrm{He}-{ }^{3} \mathrm{He}$ atom cross-section $\sigma(Q)$, shown in Fig. 5. Two contributions to a width generally contribute to the total in a very non-linear way, often as a sum of squares. Thus the contribution of a larger doppler width in ${ }^{3}$ He and smaller amplitude oscillations in $\Gamma_{0}(Q, w)$ leads to very small, probably unobservably small, oscillations in $w(Q) / Q$.

The magnitude of the calculated $W(Q) / Q \approx 2.0$ meV $\AA$ in ${ }^{3} \mathrm{He}$ agree well with the magnitudes observed by Mook ${ }^{10}$ and by Sokol et al. 31 However, we 
find the second moment $(10)$ of our calculated $S(Q, w)$ is much greater than that given by the "Gaussian" value (11). Indeed, our calculated $S(Q, w)$ have high frequency tails at large $\left(\omega-\omega_{R}\right)$ which makes $\sigma^{2}$ in (10) significantly larger than suggested by (11). Thus the values of $\langle\mathrm{KE}\rangle$ obtained by Mook and Sokol et al using (11) almost certainly underestimates the $\langle K E\rangle$. Their values lie below the most reliable Monte Carlo values. 33 A paper on this work is in preparation.

\section{PhONONS in MONOLAYERS}

The dynamics of atoms and phonons in two dimensional (2-D) solids can now be studied experimentaly.34-40 The 2-D solid is usually a monolayer or several layers of atoms absorbed on a substrate. Phonons can be observed in layers by means of inelastic scattering of neutrons, 34,35 of the atoms $36,37,38$ and of electrons. 39,40

We 41 have begun a study of phonons in 2-1 solids and monolayers. The aim is to calculate phonon energies and lifetimes, to assess anharmonic contributions and quantum effects, to determine mean-square vibrational amplitudes of the atoms and other dynamical properties of 2-D solids. A secondary aim is to study phase transitions as a function of temperature and pressure in monolayers on substrates, although there has already been substantial progress in this area. To date, this work has been done in collaboration with Ken Moleko and Bela Joos (Univ. of Ottawa) and Toufic Hakim and Siu-Tat Chui (Delaware). 
To begin we have calculated the phonon energies of the in plane modes of "floating" monolayers of Ne, Ar, Kr and Xe. By "floating" we mean an ideal 2-D monolaver with no substrate (i.e., no inteaction of the atoms with the substrate). These floating monolayers represent ideal 2-D systems essentially "floating" in space. We assume a periodic $\sqrt{3} \times \sqrt{ } 3$ structure. These "floating" monolayers are said to be "commensurate" since this is the structure an adsorbed monolayer assumes when it is commensurate with a graphite substrate.

The phonon energies have been evaluated ${ }^{4}$ within the self-consistent harmonic approximation ( $\mathrm{SCH}$ ) and in the harmonic approximation (QH). The difference between the phonon energies in these two approximations is a very direct measure of anharmonic effects.42 In the SCH Model, the vibrational amplitudes of the atoms and the phonon energies are determined iteratively until self-consistent. In Fig. 6 we show the "in plane" phonon frequency dispersion curves calculated in the SCH and HA for Xe monolayers, compared with the frequencies calculated by Marchese et al 43 using mulecular dynamics methods. The MD values include the interaction of the Xe atoms with a graphite substrate. Comparing the $\mathrm{SCH}$ and $\mathrm{QH}$ we see anharmonic effects are small. Also the interaction with the substrate affects the in plane phonon energies little, except at the Brillouin zone center where it introduces a gap. 


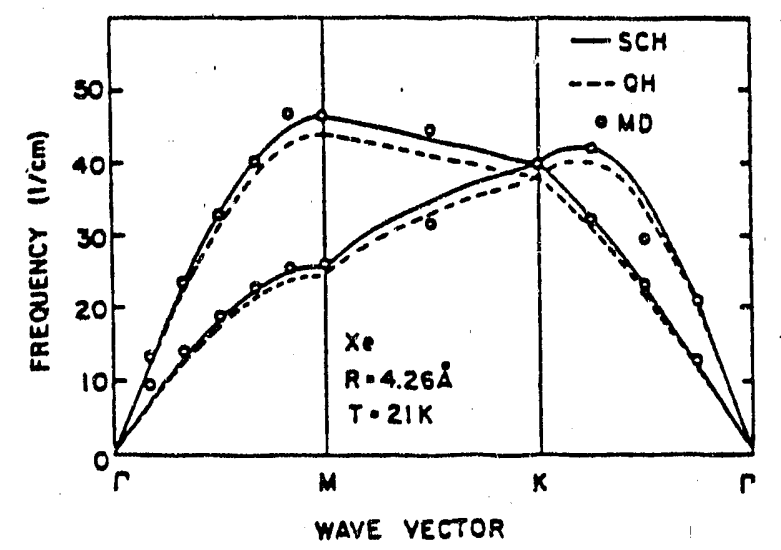

Fig. 6

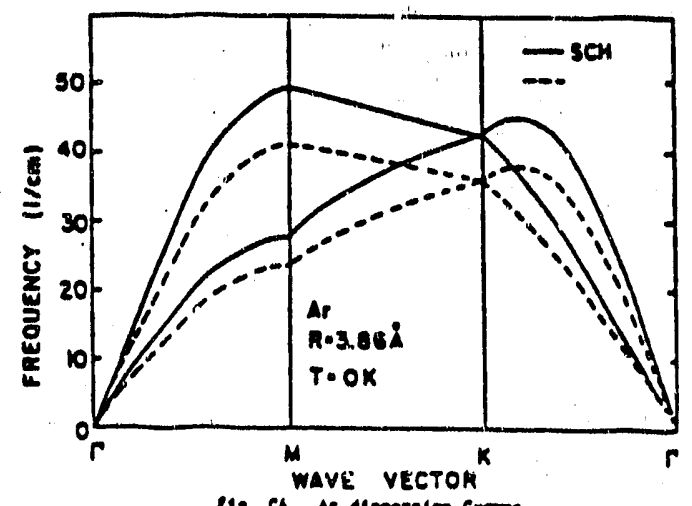

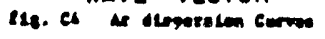

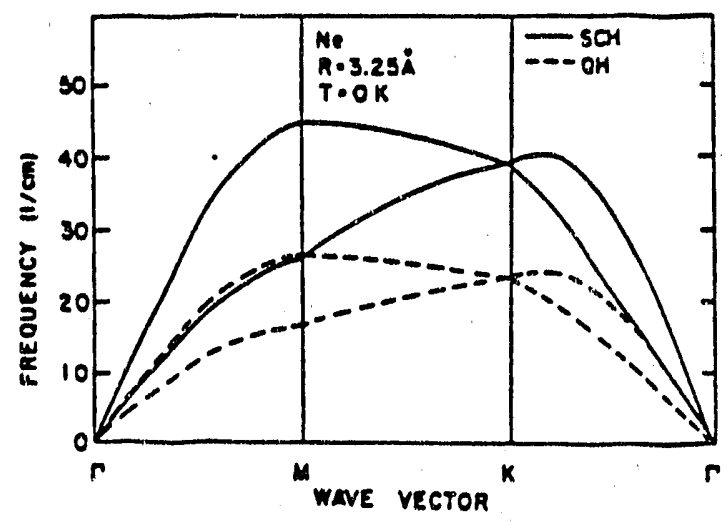

Fig. 7

Fig. 6: Phonon Dispersion Curves in 2-D Xe. 0-Monte Carlo (From Moleko et al, Ref. 41).

Fig. 7: Phonon Dispersion Curves in 2-D Ne and Ar. (From Moleko et al, Ref. 4i).

In Fig. 7 we show similar dispersion curves for 2-D Ar and Ne. There we see a substantial difference between the $\mathrm{SCH}$ and the $\mathrm{QH}$ values. This shows that anharmonic contributions are important in these 2-D solids for spacings appropriate for absorbed films on graphite. Particularly in Ne the anharmonic terms in the SCH theory increase the phonon energies by a factor of nearly 2 . 
The RMS vibrational amplitude, $\left\langle u^{2}\right\rangle \frac{1}{2}$, at $T=0 k$ is $\sim \%$ of the interatomic spacing, $R$, in xe and $\sim 8 \%$ of $R$ in Ne. While $\left\langle u^{2}\right\rangle$ is infinite at $T>0 \mathrm{~K}$, it is finite at $\mathrm{T}=0 \mathrm{~K}$ in $2 \mathrm{D}$. This is typical of the ratio $\left\langle u^{2}\right\rangle: R$ in bulk solids. The Debye temperatures calculated using the SCH frequencies are $\theta_{D} \sim 65 K$ in all of $\mathrm{Ne}, \mathrm{Ar}, \mathrm{Kr}$ and $\mathrm{Xe}$. Since the SCH theory overastimates the phonon energies ( $\left.\omega_{q} \lambda\right)$, these $\theta_{\mathrm{D}}$ may be somewhat too large in $\mathrm{Ne}$ and $\mathrm{Ar}$. These ${ }_{\mathrm{D}} \mathrm{D}$ values show that quantum effects will be important up to $\mathrm{T} \sim \theta_{\mathrm{D}} / 2 \sim 30 \mathrm{~K}$ in each case.

\section{Review Articles}

Two review articles have been written on neutron studies of liquid and solid helium: (1) Glyde, Dvnamic Properties of Quantum Solids and Fluids, Chapter 5 in Condensed Matter Research Using Neutrons ed. Lovesey and Scherm, Nato ASI Series, Vol. 112 (Plenum, 1984); (2) Glyde and Svenson, Liquid and Solid Helium, Chapter 13 in Neutron Scattering in Condensed Matter Research ed. Price and Sköld (Acidemic Press,1987). 


\section{REFERENCES}

1. A. Miller, D. Pines and P. Nozières, Phys. Rev. 127, 1452 (1962).

2. P. C. Hohenberg and P. M. Platzman, Phys. Rev.152, 198 (1966).

3. H. A. Mook, Phy. Rev. Lett. 32, 1167 (1974); Phys. Rev. Lett. 51, 1454 $(1983)$.

4. L. J. Rodriguez, H. A. Gersch, and H. A. Mook, Phys. Rev. A 9, 2085 (1974).

5. A. D. B. Woods and V. F. Sears, Phys, Rev. Lett. 39, 415 (1977).

6. V. F. Sears, E. C. Svensson, P. Martel and A. D. B. Woods, Phys. Rev. Lett. $49,279(1982)$.

7. V. F. Sears, Phys. Rev, B 30, 44 (1984).

8. E. C. Svensson and V. F. Sears, Physica 137B, 126 (1986),

9. P. Martel, E. C. Svensson, A. D. B. Woods, V. F. Sears, and R. A. Cowley, J. Low Temp. Phys. 23, 285 (1976).

10. H. A. Mook, Phys. Rev. Lett. 55,2452 (1985).

11. G. F. Chew, Phys. Kev, 80, 196 (1950).

12. J. W. M. DuMond, Rev. Mod. Phys. 5, 1 (1933).

13. Compton Scattering, edited by B. Williams (McGraw-Hill, New York, 1977).

14. V. F. Sears, Phys. Rev. 185, 200 (1969).

15. J. J. Weinstein and J. W. Negele, Phys. Rev.Lett. 49, 1016 (1982).

16. T. R. Kirkpatrick, Phys. Rev. B30, 1266 (1984).

17. G. Reiter and R. Becher, Phys. Rev. B32, 4492 (1985).

18. J. M. F. Gunn and M. Warner, Z. Yhys. B 56, 13 (1984). 
19. G. Reiter and R. Silver, Phys. Rev. Lett. 54, 1047 (1985).

20. H. R. Glyde, J. Low Temp. Phys. 59, 561 (1985).

21. B. Tanatar, G. C. Lefever and H. R. Glyde, J. Low Temp. Phys, 62, 489 (1986).

22. P. Platzman and N. Tzoar, Phys. Rev. B30, 6397 (1984).

23. H. R. Glyde (preprint).

24. B. Tanatar, G. C. Lefever and H. R. Glyde, in "Neutron Scattering" ed. G. H. Lander and R. A. Robinson (North Holland, Amsterdam, 1986).

25. G. B. West, Phys. Rep, 18C, 263 (1975).

26. L. K. Moleko and H. R. Glyde, Phys. Rev. Lett. 54, 901 (1985).

27. V. F. Sears, Solid State Comm. 11, 1307 (1972).

28. H. R. Glyde, Can. J. Phys. 52, 2281 (1974).

29. F. Family, Phys. Rev. Lett. 34, 1374 (1975).

30. v. Wong, Phys. Lett. 61A, 455 (1977).

31. P. E. Sokol, K. Sköld, D. L. Price, and R. Kleb, Phys. Rev. Lett. 54, $909(1985)$.

32. R. A. Aziz, V. P. S. Nain, J. S. Carley, W. L. Taylor and G. T. McConville, J. Chem. Phys. 70, 4330 (1979).

33. J. Carlson et al, Phys. Rev. Lett. 55, 2376 (1985).

34. H. Taub, K. Carneiro, J. K. Kjems, L. Passell and J, P. McTague, Phys. Rev. B16, 4551 (1977).

35. C. Tiby and H. J. Lauter, Surf. Sci. 117, 277 (1982).

36. K. D. Gibson and S. J. Sibener, Phys. Rev. Lett. 55, 1514 (1985); T. H. Ellis, G. Scoles; U. Valbusa, Chem. Phys. Lett, 94,247 (1983). 
37. G. Brusdeylins, R. B. Doak, and J. P. Toennies, Phys. Rev. Lett. 46 , 1138 (1980); G. Brusdeylins, R. B. Doak, and J. P. Tonnies, Phys. Rev. B27, $3662(1983)$.

38. J. F. Toennies, J. Vac. Sci. Technol. A2, 1055 (1984).

39. S. Lehwald, J. M. Szeftel, H. Ibach, T. S. Rahman, and D. L. Mills, Phys. Rev. Lett. 50, 518 (1983).

40. J. M. Szeftel, S. Lehwald, H. Ibach, T. S. Rahman, J. E. Black, and D. L. Mills, Phys, Rev, Lett. 51, 268 (1983).

41. L. K. Moleko, B. Joos, T. Hakim, H. R. Glyde and S. T. Chui, F'hys. Rev. B 34, $2815(1986)$.

42. H. R. Glyde and M. G. Smoes, Phys. Rev. B22, 6391 (1980).

43. M. Marchese, A. Jarucci and M. L. Klein, Surf. Sci. 14, 364 (1984). 
III. Publications and Presentations

\section{SCIENTIFIC PUBLICAIIONS (1986)}

1. BAND TAILS. IN DISORDERED SYSTEMS

W. Sritrakool, V. Sayakanit and H. R. Glude

Phys. Rev. B33, $1199^{\circ}$ (1986)

2. SPIN-POLARIZED DEUTRIUM

H. R. Glyde and S. I. Hernadi

IX International Workshop on Condensed Matter Theories, San Fransisco, CA, August, 1985 edited by F. B. Malik (Plenum, NY, 1986)

3. MOMENTUM DIUTRIBLTIONS AND THE IMULLSE APQROXIYATION IN HELILM

B. Tanatar. G. C. Lefever and H. R. Glyde

J. Low Temp. Phivs. 6?, 489 (1986)

4. SPIN-POLARIZED DELTRILY

H. R. Glyde and S. I. Hernadi

In Condensed Yatter Theories, Vol. 1 . edited by F. B. Malik, P. 115

(Plenum, NY, 1986)

5. SOLID AVD LIOUIID HELIUM

H. R. Glyde and E. C. Svensson

Chapter i 3 in "lieutron Scattering in Condensed Matter

Research" Ed. K. Sköld and D. L. Price (Academic Press, 1986)

6. DYNAMICS OF RARE-GAS FLOAIING MONOLAYERS

I. THE SELF-CONSISTENT PHONON THEORY

L. K. Moleko, B. Joos, T. M. Hakim, H. R. Glyde and

S. T. Chui

Phys. Rev. B344, 2815 (1986)

7. URBACH TAILS AND DISORDER

V. Sa-yakanit and H. R. Glude

Comments in Conds. Mat. Phys. (submitted)

8. IMPULSES AND EXCITATIONS IN QUANILM FLUIDS

AND SOLIDS

H. R. Glyde

Ann. Phys. (submitted)

9. HIGH MOYENTLY EXCITATIONS IN QUANTUM FLUIDS

B. Tanatar, E. F. Ialbot and H. R. Glyde

Phys. Rev. Lett. (submitted) 
POLICY PUBLICATIONS (1986):

1. INSTIIUTIONAL LINKS: AN EXAMPLE IN SCIENCE AND TECHNOLOCY H. R. Glyde and V. Sa-yakanit

horking paper, Institute for Interrational Development and Cooperation, University of Ottawa

Higher Education in Europ. (CEPES, UNESCO) Vol. 10, No. 4 p. 51 (1985)

An abbreviated version as Invited Editoral in

J. Sci. Soc. Thailand 12,61 (1986)

\section{CONFERENCE PAPERS AND SEMINARS $(1986)$}

Contributed Paper; International Conference on

iiquid and Solid Helium, Banff Canada

October 1986

Seminars and Lectures:

Impulse Appraximation in Condensed Helium

Rutherford-Appleton Laboratory

July 1986

oxtord, England

Many-Body Theory and Quantum Liquids

June 1986

Institute for Theoretical Physics

Trieste, Italy

Urbach Tails in Disordered Systems

CNRS

Grenoble, France

Neutron Scattering from Quantum Fluids

May 1986

Physik Technische Bundesanstalt

Braunschweig, Germany

Neutron Scattering frow Quantur Fluids

May 1986

University of Erlangen

June 1986

Erlangen, Cermany

Excitations in Quantum Liquids and Solids

Institute Laue Langevin

February 1986

Grenoble, France 

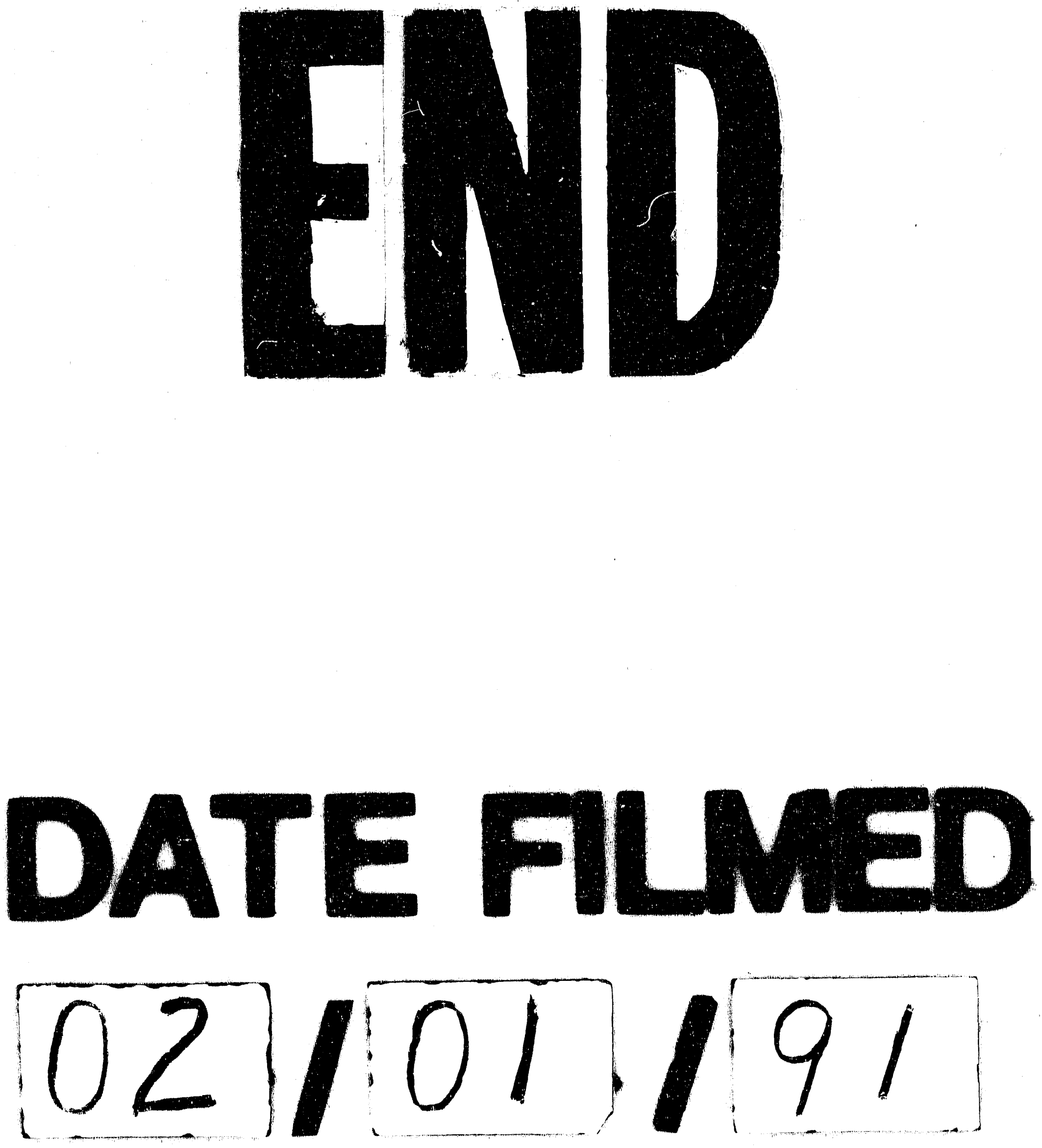
